\title{
Maternal hypertension and neurodevelopmental outcome in very preterm infants
}

\author{
Peter H Gray, Michael J O’Callaghan, Heather A Mohay, Yvonne R Burns, James F King
}

\begin{abstract}
Aim-To determine the outcome of preterm infants born to mothers with hypertension during pregnancy, and preterm controls.

Methods-107 infants of 24-32 weeks gestation, born to hypertensive mothers, and 107 controls matched for gestational age, sex, and multiple pregnancy, born to normotensive mothers, were prospectively enrolled over 2 years. Information on maternal complications and medication was obtained and neonatal mortality and morbidities recorded. Survivors were followed up to at least 2 years, corrected for prematurity.

Results-One third of the hypertensive mothers were treated with antihypertensive drugs, while $18 \%$ received convulsion prophylaxis with phenytoin. Magnesium sulphate was not prescribed. Both groups had a mean gestational age of 29.9 weeks, with the study infants having a significantly lower birthweight than the controls. Four study and three control infants died in the neonatal period. Cerebral palsy was not diagnosed in any infant of a hypertensive mother compared with five of the controls. The mean general quotient for the two groups was very similar and no difference in the incidence of minor neuromotor developmental problems was shown.

Conclusions-Maternal hypertension seems to protect against cerebral palsy in preterm infants without increasing the risk of cognitive impairment. This was independent of the use of maternally administered magnesium sulphate.

(Arch Dis Child Fetal Neonatal Ed 1998;79:F88-F93)
\end{abstract}

Department of Neonatology, Mater Misericordiae Hospitals,

Raymond Terrace

South Brisbane,

Queensland 4101,

Australia

P H Gray

Growth and

Development Clinic

P H Gray

M J O'Callaghan

H A Mohay

Y R Burns

Mater Perinatal

Epidemiology Unit

J F King

Correspondence to:

Dr P H Gray.

Accepted 16 February 1998 most women with pre-eclampsia were treated with magnesium sulphate, which may have had a beneficial, protective effect. ${ }^{7}$

The outcomes of cohorts of infants born to mothers with hypertension during pregnancy have been somewhat variable. There are reports of a higher rate of neurodevelopmental problems $^{8-10}$ in the infants of hypertensive mothers, while a large regional study found similar rates of disability for infants of mothers with or without hypertension during pregnancy. ${ }^{11}$

This study aimed to evaluate neonatal morbidity and the long term outcome of very preterm infants born to mothers whose pregnancy was complicated by hypertension, and to determine and compare the outcome in a control group of infants, born to normotensive mothers, who were matched for gestational age.

\section{Methods}

This prospective cohort study was performed over 2 years from January 1992 to December 1993 at the Mater Mothers' Hospital, Brisbane, Australia, and approved by the hospital's ethics committee. All live born infants delivered between 24 and 32 weeks gestation to mothers who were hypertensive during pregnancy were studied. Hypertension in pregnancy was diagnosed from a systolic blood pressure of $\geqslant 140$ $\mathrm{mm} \mathrm{Hg}$ and/or diastolic blood pressure $\geqslant 90$ $\mathrm{mm} \mathrm{Hg}$, or a rise in systolic blood pressure of $\geqslant 25 \mathrm{~mm} \mathrm{Hg}$ and/or a rise in diastolic blood pressure of $\geqslant 15 \mathrm{~mm} \mathrm{Hg}$ from blood pressure recordings in the first trimester. The blood pressure was confirmed by two readings 6 hours apart, except when delivery took place less than 6 hours after the abnormal reading. Classification of the hypertension was according to the Consensus Statement of the Australasian Society for the Study of Hypertension in Pregnancy ${ }^{12}$-namely: (1) pre-eclampsia, mild or severe; (2) chronic hypertension, essential or secondary; (3) pre-eclampsia superimposed on chronic hypertension.

Pre-eclampsia was defined as hypertension developing after 20 weeks gestation in the absence of a history of hypertension or renal disease. Criteria for classification as severe preeclampsia required one or more of the following: (1) blood pressure $\geqslant 170 \mathrm{~mm} \mathrm{Hg}$ systolic and/or $\geqslant 110 \mathrm{mmHg}$ diastolic; (2) proteinuria $>300 \mathrm{mg}$ per day or $\geqslant$ " $2+$ " on dipstick testing; (3) hepatic dysfunction with either epigastric pain or raised serum bilirubin and/or transaminase activities; (4) neurological symptoms of persistent headaches or visual disturbances or signs of hyper reflexia and/or clonus; 
(5) haematological disturbances of thrombocytopenia, disseminated intravascular coagulopathy, or haemolysis. Chronic hypertension was diagnosed in those women who had a history of hypertension before pregnancy or their first occurrence during the first half of the pregnancy. Superimposed pre-eclampsia was diagnosed by proteinuria and/or hyperuricaemia.

Infants of hypertensive mothers were matched to the infants of normotensive mothers using the hospital's neonatal database. Each control infant, the nearest subsequent birth to that of the index case (all within six months), was matched for gestational age ( \pm 1 week), sex, and multiple pregnancy. Gestational age was established by an ultrasound examination performed at $<20$ weeks gestation, or when this was unavailable, by the date of the last menstrual period. Selection of controls was made with no knowledge of perinatal events except that the mother was normotensive during the pregnancy. The primary cause for the preterm delivery for the control infants was documented.

Maternal characteristics, perinatal data, and details of the neonatal course of the infants were obtained from computer records and medical charts. Maternal data were collected on pregnancy and delivery as well as medications received before delivery. Infant data recorded at birth included birthweight, growth according to gestational age (Kitchen intrauterine growth charts $^{13}$ ), and Apgar scores. Details of respiratory distress syndrome (RDS) were obtained, with neonatal complications including patent ductus arteriosus (PDA), necrotising enterocolitis (NEC), sepsis and bronchopulmonary dysplasia (BPD); supplemental oxygen requirements persisting beyond 36 weeks gestational age ${ }^{14}$ were noted. Periventricular haemorrhage $(\mathrm{PVH})$, ventricular dilatation, and cystic periventricular leucomalacia (PVL) were recorded on the results of cranial ultrasonography, as described before. ${ }^{15}$ Mortality to hospital discharge was also documented.

FOLLOW UP

Following hospital discharge, infants were examined in the Growth and Development Clinic at the Mater Children's Hospital at 4, 8, 12 and 24 months after the expected date of delivery.

\section{Medical examination}

A medical history was recorded by a paediatrician at the clinic visits. A medical examination assessment, including a detailed neurological examination, was performed with no knowledge of the mother's blood pressure during pregnancy. Cerebral palsy was diagnosed on the basis of this examination and standard criteria-namely, persistent abnormality of movement and posture resulting in impairment of function, due to a non-progressive lesion of the immature brain. ${ }^{16}$ The type and severity of cerebral palsy were recorded. Mild cerebral palsy implied only slight interference with normal activities in children who were walking at 2 years, while those with more severe cerebral palsy were considered unlikely ever to walk.

\section{Neurosensory motor developmental assessment} (NSMDA) ${ }^{17} 18$

The NSMDA is a criterion referenced test and was conducted by a physiotherapist. The test evaluates the infant in terms of quality of performance as well as age appropriate task achievement. The test has six subscales: gross motor, fine motor, neurological, primitive reflexes, postural reactions and sensory motor. Each of the subscales are scored in terms of functional grades: 1 is within normal range; 2 is slight deviation with no effect on function; 3 is mild to moderate deviation with some effect on function; 4 is definite deviation limiting function; 5 is severe or profound disability. Scores from the six subscales are summed to give an overall NSMDA score. Infants are then categorised as normal or showing minimal dysfunction, or mild, moderate, or severe disability.

\section{Griffiths' Infant Ability Scale ${ }^{19}$}

This scale provides an overall general quotient and scores for each of its five subscales (locomotor, personal/social, hearing/speech, eye/hand and performance) (GQ mean 100, SD 12). Developmental delay was classified according to the Diagnostic and Statistical Manual of Mental Disorders, ${ }^{20}$ diagnostic criteria: borderline developmental delay, a GQ of between -1 and -2 SD (76-87); mild developmental delay, a GQ between -2 and -3 SD (64-75); moderate to severe developmental delay, a GQ of more than $-3 \mathrm{SD}(<64)$.

\section{STATISTICS}

Based on previous reports of impaired neurodevelopment in infants of hypertensive mothers, it was estimated that the mean GQ on the Griffiths scale would be in the range 94-95 in the study infants and about 101 for the control infants. Assuming a power of $80 \%$ and a significance of $5 \%$ (two tailed), it was estimated that 130 infants (65 study, 65 controls) would need to be followed up after discharge. It was anticipated that 170 infants of 24-32 weeks gestation would be enrolled over two years and hence it was decided to carry out the study during that time frame.

Results of categorical variables were compared using the $\chi^{2}$ and Fisher's exact tests where appropriate. Continuous normally distributed variables were examined with Student's $t$ test. The strength of association between infants of hypertensive mother and adverse outcomes was measured by the odds ratio (OR) and its 95\% confidence interval (CI). Analysis was performed using Statistix 4.0 .

\section{Results}

The study population initially consisted of 110 infants born to hypertensive mothers with 77 singletons, 15 sets of twins and one set of triplets. This represented $19.3 \%$ of the 570 live born infants of 24-32 weeks gestation delivered during the two years of the study. No con- 
Table 1 Maternal and obstetric characteristics of hypertensive and normotensive control mothers

\begin{tabular}{|c|c|c|c|}
\hline & Maternal hypertension $(n=107)$ & Controls $(n=107)$ & Significance \\
\hline Maternal age (years) ${ }^{\star}$ & $27.7(5.6)$ & $28.0(5.2)$ & NS \\
\hline Primigravida (\%) & $53(50)$ & $26(20)$ & $\mathrm{p}=0.0001$ \\
\hline Antenatal corticosteroids (\%) & $80(75)$ & $80(75)$ & NS \\
\hline Gestational age (weeks) ${ }^{\star}$ & $29.9(2.1)$ & $29.9(2.0)$ & NS \\
\hline \multicolumn{4}{|l|}{ Presentation } \\
\hline Vertex (\%) & $86(80)$ & $77(72)$ & $\mathrm{p}=0.31$ \\
\hline Breech (\%) & $18(17)$ & $27(26)$ & \\
\hline Transverse (\%) & $3(3)$ & $3(3)$ & \\
\hline \multicolumn{4}{|l|}{ Delivery } \\
\hline Vaginal (\%) & $10(9)$ & $50(47)$ & $\mathrm{p}=0.0001$ \\
\hline Casarean section (\%) & $96(91)$ & $57(53)$ & \\
\hline
\end{tabular}

* Mean (SD); NS = not significant.

trol infants of similar gestational age could be found for the triplets so they were not included in the analysis. Accordingly, 107 gestational age matched controls were identified. According to the classification of the Australasian Society for the Study of Hypertension in Pregnancy, $93(88 \%)$ infants were born to mothers with pre-eclampsia (mild 14, severe 79), 8 $(7 \%)$ infants to mothers with chronic hypertension, and $6(6 \%)$ infants to mothers with pre-eclampsia superimposed on chronic hypertension. The primary causes of preterm delivery for the infants delivered to the normotensive mothers was ante-partum haemorrhage $(n=20,19 \%)$, preterm premature rupture of membranes $(n=48,46 \%)$, preterm labour $(n=$ $33,29 \%)$, and other complications $(n=6,6 \%)$.

MATERNAL AND OBSTETRIC CHARACTERISTICS Maternal age was similar in both the hypertensive and normotensive mothers, while there were significantly more primigravid hypertensive mothers (table 1) Antenatal corticosteroids were given frequently to both hypertensive and normotensive mothers with no significant differences evident. Anti-hypertensive medications were administered to 35 (33\%) hypertensive mothers, with many having more than one anti-hypertensive agent, including hydralazine $(n=25)$, methyldopa $(n=16)$, labetolol $(n=11)$, nifedipine $(n=7)$. Nineteen mothers received convulsion prophylaxis with phenytoin. Treatment with magnesium sulphate was not used during the study. The mode of presentation at delivery was the same in both groups, though significantly more infants of hypertensive mothers were delivered by Caesarean section.

NEONATAL CHARACTERISTICS

Birthweight was significantly lower in the infants of hypertensive mothers than in the control group (table 2) Significantly more of the study infants were small for gestational age. The condition at birth, as reflected by the Apgar scores, was similar in both groups. There was an increased incidence of RDS in the infants of hypertensive mothers, though no differences were present for PDA, NEC, septicaemia and BPD. The incidence of PVH was significantly lower in the study infants, and even though the incidence of ventricular dilatation and cystic PVL was also lower, this was not significant.

Four of the study infants and three controls died before hospital discharge: survival rates in the two groups were $96.3 \%$ and $97.2 \%$, respectively. There was one intrauterine fetal death at 28 weeks gestation to a hypertensive mother, with an overall perinatal mortality of 45.0/1000 for mothers with hypertension who delivered at 24-32 weeks gestation. The cause of death for the study infants was NEC ( $n=3)$ and severe $\operatorname{BPD}(n=1)$, while the three control infants all died within six hours of birth with severe RDS. One infant also had clinical pulmonary hypoplasia secondary to prolonged premature rupture of the membranes.

\section{FOLLOW UP}

Two study infants died from sudden infant death syndrome before the four month clinic visit. Follow up to at least 2 years was obtained for $97(96 \%)$ of the 101 long term survivors. Two infants never returned for follow up, while one could not be contacted after the 4 month clinic visit. One child was seen at 1 year, corrected for prematurity, at which time he was developmentally normal with normal Griffiths scores, but failed to attend for the two year clinic visit. These four children were excluded from the analysis. Four other children were assessed by local paediatricians, while a further two were assessed at home by a paediatrician

Table 2 Neonatal characteristics of study and control infants

\begin{tabular}{llll}
\hline & Study $(n=107)$ & Controls $(n=107)$ & Significance \\
\hline Birthweight $(\mathrm{g})^{\star}$ & $1233(376)$ & $1469(414)$ & $\mathrm{p}=0.0001$ \\
Small for gestational age (\%) & $14(13)$ & $2(2)$ & $\mathrm{p}=0.002$ \\
Apgar score & & & $\mathrm{NS}$ \\
$\quad 1$ min $^{\star}$ & $5.8(2.3)$ & $5.7(2.5)$ & $\mathrm{NS}$ \\
$\quad$ min & $8.5(1.2)$ & $8.3(1.6)$ & $\mathrm{p}=0.04$ \\
Respiratory distress syndrome (\%) & $58(54)$ & $42(39)$ & $\mathrm{NS}$ \\
Patent ductus arteriosus (\%) & $15(14)$ & $18(17)$ & $\mathrm{p}=0.02$ \\
PVH & $2(2)$ & $11(10)$ & $\mathrm{NS}$ \\
$\quad$ Grade 1-4 (\%) & 0 & $3(3)$ & $\mathrm{NS}$ \\
$\quad$ Grade 3, 4 (\%) & $2(2)$ & $5(5)$ & $\mathrm{NS}$ \\
Ventricular dilitatation & 0 & $2(2)$ & $\mathrm{NS}$ \\
Periventricular leucomalacia (\%) & $4(4)$ & $3(3)$ & \\
Mortality (\%) & & & \\
\hline
\end{tabular}

* Mean (SD); NS = not significant; $\mathrm{PVH}=$ periventricular haemorrhage. 
Table 3 Developmental status of infants of hypertensive mothers and controls

\begin{tabular}{lll}
\hline & $\begin{array}{l}\text { Study infants } \\
(n=96)\end{array}$ & $\begin{array}{l}\text { Control infants } \\
(n=101)\end{array}$ \\
\hline Normal (\%) & $80(83)$ & $88(87)$ \\
Borderline developmental delay (\%) & $12(13)$ & $10(10)$ \\
Mild developmental delay (\%) & $2(2)$ & $1(1)$ \\
Moderate/severe developmental delay (\%) & $2(2)$ & $2(2)$ \\
\hline
\end{tabular}

Table 4 Grades of dysfunction on neurosensory motor developmental assessment of study and control infants at 2 years

\begin{tabular}{lcc}
\hline & $\begin{array}{l}\text { Study infants } \\
(n=90)\end{array}$ & $\begin{array}{l}\text { Control infants } \\
(n=90)\end{array}$ \\
\hline Normal (\%) & $78(81)$ & $70(78)$ \\
Minimal dysfunction (\%) & $12(13)$ & $16(18)$ \\
Mild dysfunction (\%) & $5(6)$ & $4(4)$ \\
\hline
\end{tabular}

and a psychologist and thus no NSMDA was performed.

For the control infants, information concerning their outcome to at least 2 years was available for $102(98 \%)$ of the 104 long term survivors. Two infants were assessed at the age of 1 year (at which stage they were developmentally normal), but subsequently became untraceable. Three infants were assessed by interstate paediatricians, with nine infants being examined at home, and NSMDA available for 90 infants.

\section{NEURODEVELOPMENTAL OUTCOME}

The five children with cerebral palsy had one of the spastic syndromes and all belonged to the control group, with none of the infants of hypertensive mothers displaying features of cerebral palsy (Fisher's exact test, $\mathrm{p}=0.04$ ). Four children had mild spastic diplegia and one severe diplegia. The remaining child had severe spastic quadriplegia.

To examine whether the association of cerebral palsy occurring in the control group was independent of other possible antenatal risk factors, stratified analyses were undertaken, with premature rupture of membranes (PROM) and chorioamnionitis as independent variables. Three infants in the hypertensive group were born after PROM, with no cases of cerebral palsy being diagnosed. In the control group, however, cerebral palsy occurred in four of the $46(8.7 \%)$ infants born after PROM compared with one of the other $55(1.8 \%)$ control infants; clinical chorioamnionitis was not present in any of the hypertensive pregnancies compared with 10 of the control pregnancies. Cerebral palsy was diagnosed in two of the $10(20 \%)$ infants compared with 3 cases of the $91(3.3 \%)$ infants born without this pregnancy complication. Neither of these associations was significant.

As PVH is associated with the development of cerebral palsy, a stratified analysis was performed omitting the three infants with grade 3-4 haemorrhage, all of whom were in the control group. For children without grade 3-4 PVH, three of the 98 controls had cerebral palsy compared with none of the 96 study infants; this association was not significant. Although there were significantly more vaginal deliveries in the control group than in the study infants, the risk of cerebral palsy was independ- ent of the mode of delivery. For the control infants, two of $50(4 \%)$ delivered vaginally developed cerebral palsy compared with three of the $54(5.3 \%)$ infants delivered by caesarean section.

Analysis of the results of the psychometric testing showed an almost identical mean score of 96.9 (SD 11.5) in the infants of hypertensive mothers compared with 96.5 (SD 11.1) in the controls $(p=0.92)$. Furthermore, no significant differences were seen on analysis of the Griffiths subscales between the groups. Table 3 shows that while the incidence of developmental delay was slightly higher in the study infants $(17 \%)$ than in the controls $(13 \%)$, but this was not significant. (OR 1.33; 95\% CI 0.61-2.99). To establish the influence of the severity of maternal hypertension on outcome, infants born to mothers with severe pre-eclampsia were compared with their controls. No differences in the GQs were found (study group mean GQ 96.9, SD 11.4; controls group mean GQ 95.6, SD 11.5).

As minor neuromotor developmental problems, especially in the area of fine motor and visuomotor perception, have been reported in the infants of hypertensive mothers, we compared the scores on the NSMDA for our study infants with the controls, having excluded the infants diagnosed with cerebral palsy. Table 4 shows no increase in the incidence of minimal or mild dysfunction in the infants of hypertensive mothers (OR 0.82; 95\% CI 0.40- 1.68). Additionally, when the subscales on the NSMDA were analysed, no statistical differences were found between the two groups. In the area of sensory-motor performance, however, $19 \%$ of the study infants deviated from normal compared with $11 \%$ of the controls (OR 1.86; 95\% CI $0.80-4.33, \mathrm{p}=0.14$ ).

\section{Discussion}

The survival rate to hospital discharge of very preterm infants born to hypertensive mothers was high $(96.3 \%)$, and as there was only one intrauterine fetal death, the perinatal mortality was low. Indeed, the perinatal mortality compared very favourably with other reports of similar populations of preterm infants born to hypertensive mothers, ${ }^{20}{ }^{21}$ and reflects a high overall standard of perinatal care. Significantly more of the hypertensive infants were delivered by caesarean section, with most of these being carried out before the onset of labour. Even though it was not always clear whether the delivery was performed primarily in either the maternal or fetal interest, it seemed that most deliveries were by caesarean section before the onset of labour because of deterioration in maternal health. Obstetricians vary widely in their use of caesarean section as the indicated mode of delivery for mothers whose pregnancies are complicated by hypertension, ${ }^{22}$ but recent studies have reported a caesarean section rate of $84 \%$ to $94 \%,{ }^{9-23}$ comparable with the $92 \%$ found in our investigation.

Antenatal corticosteroids were used extensively in both groups of mothers, though the incidence of RDS was significantly higher in the infants of the hypertensive mothers com- 
pared with the control group of neonates. This may have been related to the significantly higher rate of caesarean section in the hypertensive group, which has also been noted in previous reports. ${ }^{23}{ }^{24}$ Nevertheless, despite the higher incidence of RDS, the incidence of $\mathrm{PVH}$ was significantly lower in the infants of hypertensive mothers. Possibly due to low numbers, there was no significant difference in the incidence of grades 3 to $4 \mathrm{PVH}$ or PVL, but notably, no infant in the hypertension group had major haemorrhage or cystic PVL. The results of recent studies also found that maternal hypertension confers a protective effect in relation to the development of $\mathrm{PVH} .{ }^{25}{ }^{26}$ Importantly, this protection seemed to be independent of the mode of delivery. ${ }^{26}$

On follow up, no infant born to a hypertensive mother was diagnosed with cerebral palsy, suggesting that maternal hypertension has a protective effect against cerebral palsy in very preterm infants. Previous case control studies have not reported this very important finding. ${ }^{8}{ }^{9}$ Indeed, Szymonowicz and $\mathrm{Yu}^{8}$ found that the incidence of cerebral palsy was higher in infants born to mothers with pre-eclampsia, with all cases apparently being related to neonatal PVH. Spinillo et al found no difference in the incidence of cerebral palsy between infants born to hypertensive mothers and normotensive controls, though notably, in contrast to most other studies, the incidence of PVH was higher in the hypertensive group of infants.

Conflicting evidence has also come from epidemiological studies examining antenatal antecedents of cerebral palsy, with some reporting an association with maternal hypertension $^{34}$ and others unable to find any significant association. ${ }^{27} 2829$ These studies, however, have included both term and preterm infants. Studies that have specifically examined antenatal risk factors for cerebral palsy in very preterm infants have found that pre-eclampsia is associated with a decreased risk of cerebral palsy, ${ }^{5}$ a finding that is supported by our data. It is unclear why maternal hypertension/preeclampsia provides protection against cerebral palsy. Nelson and Grether ${ }^{7}$ have suggested that the use of magnesium sulphate given for tocolysis or for convulsion prophylaxis to mothers with pre-eclampsia may provide neuroprotection for the newborn infant. Because most women in North America with hypertension complicating pregnancy receive magnesium sulphate for pre-eclampsia, it has not been possible to determine whether pre-eclampsia itself or its treatment result in a decreased risk of cerebral palsy in the infants of pre-eclamptic mothers. ${ }^{6}$ Importantly, among our population no mother received magnesium sulphate for convulsion prophylaxis.

There is an association between $\mathrm{PVH}$ and cerebral palsy, and as already discussed, a reduced rate of $\mathrm{PVH}$ is associated with pre-eclampsia. Murphy et $a \bar{l}$ have proposed that this may be the mechanism by which preeclampsia confers protection against cerebral palsy. In their study, however, no data were given with regard to the incidence of neonatal morbidities including $\mathrm{PVH}$ in their group of very preterm infants. We found that following a stratified analysis with the omission of infants with grade 3-4 PVH, even though there was a trend towards an association between the beneficial effects of hypertension with regard to cerebral palsy, this was not significant. Accordingly our data indicate that maternal hypertension protects against cerebral palsy by way of a lower incidence of PVH, but a larger study with greater statistical power might show that the protection obtained is independent of the presence of PVH.

Periventricular white matter injury in preterm infants is strongly associated with the development of cerebral palsy. ${ }^{30}$ Surprisingly, a necropsy study has revealed an association between this cerebral lesion and pre-eclampsia. ${ }^{31}$ It was thus suggested that the reduced risk of cerebral palsy noted among survivors of hypertensive mothers might have been due to death of infants who would have developed cerebral palsy had they survived. Three of our four infants in the hypertensive group who died before hospital discharge had necropsy examinations. As no infant displayed evidence of PVL, it would seem that death of infants destined to have cerebral palsy was not an explanation for the findings in the present study.

The cognitive development in our investigation did not differ between the two groups of infants, in accordance with that found by Spinillo et $a l^{9}$ in their prospective case control study. Szymonowicz and $\mathrm{Yu}^{8}$ have reported a higher incidence of developmental delay in infants of hypertensive mothers, even though this was not significant. Additionally, on follow up at 4 years of age Ounsted et $a l^{32}$ found that hypertension was associated with slight developmental delay, while results from the Dundee Development Screening Programme showed that severe hypertension was associated with global intellectual delay. ${ }^{33}$ When we performed a subgroup analysis on our infants born to mothers with severe pre-eclampsia and their controls, again no differences were evident between the groups. This is important information in providing supporting evidence that delivery should not be delayed in the face of deteriorating maternal condition. It is not entirely clear why some reports have shown developmental delay in infants of hypertensive mothers. Those studies, however, were performed in infants born in the 1970s and early 1980 s, and it may be that improvements in perinatal care in the past decade have resulted in improved cognitive development for infants of hypertensive mothers.

The NSMDA can be used to evaluate infants in terms of quality of performance as well as age appropriate task achievements. The previous findings of impaired fine motor performance ${ }^{32} 34$ and sensory motor perception ${ }^{10} 34$ in infants of pre-eclamptic mothers makes the NSMDA, which has subscales including fine motor and sensory motor performance, an appropriate test instrument to assess infants of hypertensive mothers. When the control infants with cerebral palsy were excluded no increased risk of 
dysfunction could be demonstrated in the hypertensive group of infants.

In conclusion, the results of this study have shown that maternal hypertension is associated with a reduced incidence of cerebral palsy in very preterm infants. Importantly, this occurred without any increase in impaired cognitive development or minor neurological problems. The apparent protective effect of maternal hypertension has occurred without the use of magnesium sulphate. In light of recent reports, however, of a marked reduction in the risk of cerebral palsy and mental retardation in infants of mothers who received magnesium sulphate prior to delivery, ${ }^{75}$ it seems appropriate that a randomised controlled clinical trial on the prenatal use of magnesium sulphate should be undertaken.

We thank Mrs Yvonne Rogers, project coordinator and the res of the Growth and Development clinic team for their invaluable assistance with the follow-up assessments. This study was funded by the British Red Cross Society and the Alfred Juster Memorial Fund.

1 McCowan LME, Buist RG, North RA, Gamble G. Perinatal morbidity in chronic hypertension. Br F Obstet Gynaecol 1996;103:123-9.

2 Brown MA, Buddle ML. Hypertension in pregnancy: maternal and fetal outcome according to laboratory and clinical features. Med F Aust 1996;165:360-5.

3 Hagberg G, Hagberg B, Olow I. The changing panorama of cerebral palsy in Sweden 1954 - 70. III. The importance of foetal deprivation of supply. Acta Paedriatrica Scand 1976;65:403-8.

4 Jonas O, Stern LM, Macharper T. A South Australian study of pregnancy and birth risk factors associated with cerebral of pregnancy and birth risk factors associat
palsy. Int f Rehabil Res 1989;12:159-66.

5 Murphy DJ, Sellers S, MacKenzie IZ, Yudkin PL, Johnson AM. Case-control study of antenatal and intrapartum risk factors for cerebral palsy in very preterm singleton babies. factors for cerebral palsy in

6 Grether JK, Nelson KB, Emery ES, Cummins SK. Prenatal and perinatal factors and cerebral palsy in very low birth weight infants. $\mathcal{F}$ Pediatr 1996;128:407-14.

7 Nelson KB, Grether JK. Can magnesium sulfate reduce the risk of cerebral palsy in very low birthweight infants? Pediatrics 1995;95:263-9.

8 Szymonowicz W, Yu VYH. Severe pre-eclampsia and infants of very low birth weight. Arch Dis Child 1987;62:712-6.

9 Spinillo A, Iasci A, Capuzzo, E, Egbe TO, Colona L, Fazzi E. Two-year neurodevelopmental outcome after expectant management and indicated preterm delivery in hypertensive pregnancies. Acta Obstet Gynecol Scand 1994;73:625-

10 Korkman M, Hilakivi-Clarke LA, Autti-Ramo I, Fellman V, Grandstrom M-L. Cognitive impairments at two years of age after prenatal alcohol exposure or perinatal asphyxia. age after prenatal alcohol exp
Neuropediatrics 1994;25:101-5

11 Van Zeban der Aa DM, Verwey RA, Verloove-Vanhorick SP, Brand R, Ruys JH. Maternal hypertension and very preterm infants' mortality and handicaps. Eur $\mathcal{F}$ Obstet Gynecol Reprod Biol 1986;23:137-44.

12 Australasian Society for the Study of Hypertension in Pregnancy. Management of hypertension in pregnancy: executive summary. Med $\mathcal{F}$ A ust 1993;158:700-2.

13 Kitchen WH, Robinson HP, Dickinson AJ. Revised intrauterine growth curves for an Australian population. Aust Paediatr f 1983;19:157-61.
14 Shennan AT, Dunn MS, Ohlsson A, Lennox K, Hoskins EM. Abnormal pulmonary outcomes in premature infants: prediction from oxygen requirements in the neonatal period. Pediatrics 1988;82:527-32.

15 Tudehope DI, Masel J, Mohay H, et al. Neonatal cranial ultrasonography as predictor of 2 year outcome of very low birthweight infants. Aust Paediatr 7 1989;25:66-71.

16 Bax MCO. Terminology and classification of cerebral palsy. Dev Med Child Neurol 1964;6:295-307.

17 Burns YR. NSMDA. Physiotherapy assessment for infants and young children. Brisbane: Copyright Publishing Company, 1992.

18 O'Callaghan MJ, Burns Y, Gray P, et al. Extremely low birth and control infants at 2 years corrected age: a comparison of intellectual abilities, motor performance, growth and health. Early Hum Dev 1995;40:115-25.

19 Griffiths R. The ability of young children. London: Child Development Research Centre, 1970

20 APA. Diagnostic and Statistical Manual of Mental Disorders. 3rd edn (revised). Washington, DC: American Psychiatric Association, 1987:22-3.

21 Harms K, Rath W, Herting E, Kuhn W. Maternal hemolysis, elevated liver enzymes, low platelet count and neonatal outcome. Am F Perinatol 1995;12:1-6.

22 Derham RJ, Hawkins DF, de Vries LS, Aber VR. Outcome of pregnancies complicated by severe hypertension and delivered before 34 weeks; stepwise logistic regression analysis of prognostic factors. $\mathrm{Br} \mathcal{F}$ Obstet Gynaecol 1989;96:1173-81.

23 Catanzarite V, Quirk JG, Aisenbrey G. How do perinatologists manage preeclampsia? Am f Perinatol 1991;8:7-10.

24 Banias BB, Devoe LD, Nolan TE. Severe preeclampsia in preterm pregnancy between 26 and 32 weeks' gestation. Am f Perinatol 1992;9:357-60.

25 Tubman TRI, Rollins MD, Patterson C, Halliday HL. Increased incidence of respiratory distress syndrome in babies of hypertensive mothers. Arch Dis Child 1991;66:52-4.

26 Kuban KCK, Leviton A, Pagano M, Fenton T, Strassfeld R, Wolff M. Maternal toxaemia is associated with reduced incidence of germinal matrix hemorrhage in premature babies. F Child Neurol 1992;7:70-6.

27 Shankaran S, Baeur CR, Bain R, Wright LL, Zachary J. Prenatal and perinatal risk and protective factors for neonatal intracranial hemorrhage. Arch Pediatr Adolesc Med 1996;150:491-7.

28 Nelson KB, Ellenberg JH. Antecedents of cerebral palsy I. Univariate analysis of risks. $A m \quad \mathcal{F}$ Dis Child 1985;139:1031-8

29 Palmer L, Blair E, Petterson B, Burton P. Antenatal antecedents of moderate and severe cerebral palsy. Paediatr Perinat Epidemiol 1995;9:171-84.

30 Rogers B, Msall M, Owens T, et al. Cystic periventricular leukomalacia and type of cerebral palsy in preterm infants. f Pediatr 1994;125:S1-8.

31 Murphy DJ, Squier MV, Hope PL, Sellers S, Johnson A. Clinical associations and time of onset of cerebral white matter damage in very preterm babies. Arch Dis Child 1996;75:F27-F32.

32 Ounsted MK, Moar VA, Good FJ. Hypertension during pregnancy with and without specific treatment; the development of the children at four years. Brf Obstet Gynaecol 1980;87:19-24.

33 Taylor DJ, Howie PW, Davidson J, Davidson D, Drillien CM. Do pregnancy complications contribute to neurodevelopmental disability? Lancet 1985;i:713-6.

34 Martikainen A. Growth and development at the age of 1.5 years in children with maternal hypertension. F Perinat Med 1989;17:259-69.

35 Schendel DE, Berg CJ, Yeargin-Allsopp M, Boyle CA, Decourle P. Prenatal magnesium sulfate exposure and the risk for cerebral palsy and mental retardation among very low-birth-weight children aged 3 to 5 years. $\mathscr{f} A M A$ 1996;276:1805-10. 